\title{
A Risk Evaluation Algorithm of the Parameter Measurement based on Reliability Design for Ships
}

\author{
Zhang Ting*, Gu Jiali, He Menglei and Xia Jianchao \\ Wuhan Second Ship Design and Research Institute, Hubei Province, Wuhan, China \\ "zhangting200212@163.com
}

Keywords: Reliability design, Measurement risk, Evaluation model, Measurement equipment, Parameter measurement

\begin{abstract}
A new evaluation method of the parameter measurement risk based on reliability design is present in the paper. Based on the Weight Estimation and reliability model, the calculated model of measurement risk probability is described. The equipment's measurement reliability and system's reliability design are fully taken into account in this approach. It provides a theoretical support for the quantitative assessment of parameter measurement risk. And this modeling would be a helpful step to the performance valuation of measuring systems.
\end{abstract}

\section{Introduction}

The value of parameter usually is got by measurement. The error and uncertainty which is with the measurement is unequal to zero. While the nonzero error or uncertainty is more the allowable limit, a series of negative cases such as loss would be brought out. The probability of negative cases by measurement is its risk [1]. The measurement risk probability can be described by Equation 1 [2].

$$
v=1-R
$$

Where $v$ is risk probability of parameter measurement, $R$ is reliability of parameter measurement, and is probability for the value of parameter within its limits as well.

Eq. 1 shows that the risk probability could be calculated by its reliability. In "direct measurement", the reliability of parameter measurement is mainly determined by the equipments which are used to measure the parameter. But the measurement reliability is also affected by its design level with the more complicated calculation models and more reliability design. The paper gives a new algorithm for parameter measurement risk. The influences of its design factors for the parameter measurement reliability are fully considered in this paper.

The organization of this paper is as follows. Section 2 introduces the reliability formula of equipments based on Weight Estimation. The optimized reliability model of ship equipments based on reliability design is discussed in Section 3. Section 4 discusses the risk evaluation model of the parameter measurement based on reliability design for ships. Finally, Section 5 gives an example and Section 6 concludes this paper. 


\section{Reliability model of equipments based on Weight Estimation}

Reliability of test equipment can be estimated by the probability of which the parameters within the definite limits during the providing time. The reliability value will reduce over time. It is means the probability of which the parameter goes beyond the limits will increase [3]. Reliability models are reliability block diagram and mathematics models that built to predict and evaluate reliability of test equipment based on its parameters. At present, most of models are built by the distribution of its break-down. This reliability model of equipment takes full account of the influence factors to the reliability by the weight estimation method [4,5] based on its break-down distribution.

\subsection{The coefficient of the main influence factor for test equipment's reliability, $c$}

The main influence factors are features of equipment, performance specifications and its operational environment [6]. The relationship between them can be described by Equation 2 .

$$
\sum_{i=1}^{3} c_{i}+\sum_{j=4}^{n} c_{j}=1 \quad i=1,2,3 \quad j=3, \cdots, n
$$

Where $c_{1}$ is the coefficient of the feature, $c_{2}$ represents the specification and $c_{3}$ means operational environment, $\sum_{j=4}^{n} c_{j}$ includes the coefficients of the other factors, $n$ is the number of the factors. In special application, $n=3, \quad \sum_{j=4}^{n} c_{j}=0$. The number of the factors, $n$, can be more according to actual condition.

Within restriction of hypotheses, the influence of factors could be confirmed by its situations. Otherwise, the influence of all the factors is approximately the same. And the coefficients are equal.

\subsection{The weight of the main influence factor for test equipment's reliability, $w$}

Each of the factors has its own elements. Such as the features could be work principle, structure or material. The specifications include max permissible error, repeatability and stability etc. the operational environment could be environmental conditions, use frequency or maintenance. The elements are analyzed and evaluation for 0 to 1 . Lower score means the influence of the feature to the reliability more negative. The relationship between the weights of the factors can be described by Equation 3.

$$
w_{i}=\prod_{j=1}^{m} w_{i j} \quad j=1,2,3, \cdots, m
$$

Where $w_{\mathrm{i}}$ is the weight of the factor, $w_{\mathrm{ij}}$ is the value of the element, $m$ is the number of the elements for the factor.

\subsection{The reliability of test equipment, $R$}

According to the coefficients and weights above, the reliability of test equipment, $R_{0}$, can be calculated by the formula below with its distribution model of break-down character.

$$
R_{0}=\sum_{i}^{n} c_{i} w_{i} \times r(t) \quad i=1,2,3, \cdots, n
$$

Where $R_{0}$ is the reliability of test equipment, $r(t)$ is the distribution model of break-down character. Without restriction of hypotheses, $w_{\mathrm{i}}$ equals to 1 , and $R_{0}$ is as same as $r(t)$. 


\section{The optimized reliability model of ship equipments based on reliability design}

Along with the rapid development of industry, the reliability design principles have been widely used in product development. The point of these principles is finding the weaknesses or troubles of the systems from three aspects: the units, the relationship between units and environment, then taking measures to remove or improve the matters. Therefore, the widely application of reliability principles in ships has a great effect to the weights, $w$.

There are mainly three methods of design for ship reliability [7]. The first method is trouble prevention for unit, which includes the derating and margin designs. The second way is trouble prevention for system, such as the redundancy and fault-tolerant designs. The last one is environmental impact minimization, which can be the analyses of environmental loads or environment protection design [2].

\subsection{The influence of margin design to the weight of the feature $w_{1}$}

In the field of mechanical reliability design, the concept of margin is to add a safety factor which is more than 1, trying to make sure no fault happened [7]. The margin design increases the influence of the feature. And the relationship is as follow.

$$
w^{\prime}=w_{1} \times k
$$

where $w_{1}$ is the weight of the feature, $w^{\prime}{ }_{1}$ is the weight of the feature with the margin designs, and $k$ is the safety factor of which the value is more than 1 .

\subsection{The influence of derating design to the weight of the specification $w_{2}$}

For the lower basic fault rate and better work reliability, the stress of electricity and temperature for the equipment should be lower than their ratings. It is the derating design. The derating design increases the influence of the specification. And the relationship is as follow.

$$
w_{2}^{\prime}=w_{2} / \lambda
$$

Where $w_{2}$ is the weight of the specification, $w_{2}^{\prime}$ is the weight of the specification with the derating designs, and $\lambda$ is the derating factor of which the value is less than 1 .

\subsection{The influence of environment protection design to the weight of the operational environment $w_{3}$}

The environment protection design is taking measures to make sure the equipments can wok safely in multitude and complicated environmental conditions. The approach is to identify its environment loads and improve or adapt them. The environment protection design increases the influence of the operational environment. And the relationship is as follow.

$$
w_{3}^{\prime}=w_{3} \times \eta
$$

Where $w_{3}$ is the weight of the operational environment, $w_{3}^{\prime}$ is the weight of the operational environment with the environment protection designs, and $\eta$ is the environment protection degree of which the value is more than 1.

The conditions are comprehensive. So is the environment protection design. The thermal, pressure, electromagnetic and space radiation environment design are the environment protection design principles used commonly [7]. The relationship between them is as follow.

$$
\eta=\prod_{i=1}^{p} \eta_{i} \quad i=1,2,3, \cdots, p
$$


Where $\eta_{\mathrm{i}}$ is the degree of each environment protection design, $p$ is the number of environment protection design principles used.

\subsection{The reliability model of ship's system based on its reliability design}

According to the formula 5 to 8 above, the reliability model optimized is as follow $[8,9]$.

$$
R^{\prime}=\sum_{i=1}^{3} c_{i} w_{i} \times r(t)=\left[\left(c_{1} \times w_{1} \times k+c_{2} \times \frac{w_{2}}{\lambda}+c_{3} \times w_{3} \times \eta\right)+\sum_{j=4}^{n} c_{j} w_{j}\right] \times r(t)
$$

Where $R^{\prime}$ is the optimized reliability of equipment, $c_{1}$ is the coefficient of the feature for equipment, $w_{1}$ is the weight of the feature, $c_{2}$ is the coefficient of the specification, $w_{2}$ is the weight of the specification, $c_{3}$ is the coefficient of the operational environment, $w_{3}$ is the weight of the operational environment, $k$ is the safety factor of which the value is more than $1, \lambda$ is the derating factor of which the value is less than $1, \eta$ is the environment protection degree of which the value is more than $1, \sum_{j=4}^{n} c_{j} w_{j}$ is the weighted sums of other factors, $n$ is the number of the influence factors, and $r(t)$ is the distribution model of break-down character.

According to Eq. 9, without the special application, $n$ equals to $3, \sum_{j=4}^{n} c_{j} w_{j}$ is zero. $k$ equals to 1 while there is no margin design. $\lambda$ equals to 1 while there is no derating. $\eta$ equals to 1 while there is no environment protection. And without restriction of hypotheses, $w_{1}, w_{2}$, and $w_{3}$ are all equaling to 1 . Then $R$ equals to $r(t)$.

\section{The risk evaluation model of the parameter measurement based on reliability design for ships}

In the redundancy design of measurement, more than one test equipment are used to measure the value of certain parameter, for the value we get reliability and exactly. The reliability model of the measurement structure can be approximated by parallel model. The reliability model of the parameter measurement for ship system is as follow [7].

$$
R=1-\prod_{k=1}^{q}\left(1-R_{k}^{\prime}\right)
$$

Where $R$ is the measurement reliability of parameter, $R_{\mathrm{k}}^{\prime}$ is the measurement reliability of test equipment, $q$ is the number of test equipments measuring the parameter.

Assuming the equipments have the same measurement principles and are independence, the reliability model of parameter measurement is as follow.

$$
R=1-\left(1-R^{\prime}\right)^{q}
$$

According to Eq. 1 and Eq. 9, the risk probability of parameter measurement based on reliability design can be calculated by the formula below.

$$
v=\left(1-\left[\left(c_{1} \times w_{1} \times k+c_{2} \times \frac{w_{2}}{\lambda}+c_{3} \times w_{3} \times \eta\right)+\sum_{j=4}^{n} c_{j} w_{j}\right] \times r(t)\right)^{q}
$$

Where $v$ is the risk probability of parameter measurement based on reliability design, $c_{1}$ is the coefficient of the feature for equipment, $w_{1}$ is the weight of the feature, $c_{2}$ is the coefficient of the specification, $w_{2}$ is the weight of the specification, $c_{3}$ is the coefficient of the operational environment, $w_{3}$ is the weight of the operational environment, $k$ is the safety factor of which the value is more than $1, \lambda$ is the derating factor of which the value is less than $1, \eta$ is the environment 
protection degree of which the value is more than $1, \sum_{j=4}^{n} c_{j} w_{j}$ is the weighted sums of other factors, $n$ is the number of the influence factors, $r(t)$ is the distribution model of break-down character, $q$ is the number of test equipments measuring the parameter

According to Eq. 12, without the special application, $n$ equals to $3, \sum_{j=4}^{n} c_{j} w_{j}$ is zero. $k$ equals to 1 while there is no margin design. $\lambda$ equals to 1 while there is no derating. $\eta$ equals to 1 while there is no environment protection. And without restriction of hypotheses, $w_{1}, w_{2}$, and $w_{3}$ are all equaling to 1 . Then $R$ equals to $r(t)$.

\section{The example of the model}

The reliability design indexes of certain ship system are list in the table below.

Tab. 1 The index of reliability design for the parameter measurement

\begin{tabular}{|c|c|c|c|c|}
\hline No. & Reliability design principle & Reliability design index & Value & Note \\
\hline 1 & Redundancy design & $p$ & 2 & \\
\hline 2 & Derating design & $\lambda$ & 0.9 & \\
\hline 3 & Environment protection design & $\eta$ & 1.09 & \\
\hline 4 & Margin design & $k$ & 1 & \\
\hline
\end{tabular}

Assuming the test equipments for the parameter are all electronic products, the relationship between the reliability parameter of the equipment and the operating time has an exponential distribution $[10,11]$. According to Eq. 11, the risk probability of the parameter without restriction of hypotheses can be calculated as follow.

$$
v=\left(1-\left[\left(c_{1} \times w_{1} \times k+c_{2} \times \frac{w_{2}}{\lambda}+c_{3} \times w_{3} \times \eta\right)+\sum_{j=4}^{n} c_{j} w_{j}\right] \times r(t)\right)^{q}=\left(1-1.07 \mathrm{e}^{-\theta t}\right)^{2}
$$

Upon the risk probability above, the risk can be got by evaluating the degree of the risk. Analyzing the acceptability of the risk is helpful to make the measures to control the troubles. Meanwhile, the rationality of the specification of the parameter can be appraised based on the risk probability value.

\section{Conclusions}

Based on the engineering application, a risk evaluation approach focused on the parameter measurement is discussed in the paper. And the reliability model based on ship structure and the features of reliability design methods are considered in the algorithm. The engineering and reliability design factors added in the model makes it more exact and applicability. This algorithm would be a helpful step to the risk probability evaluation from different aspects. There would be further study in the evaluation method for the weight of the main influence factor.

\section{References}

[1] LI Hongli, ZHANG Yu, QIAO Yang et al. Concept, Dynamic Measurement and Application of Risk[J]. Chinese Journal of Ergonomics, 2013, 19(4): 86-90.

[2] FU Sha, SONG Dan. An Information Security Risk Assessment Method Based on AHP and Exploration in Laboratory, 2012(6):207-210.

[3] YU Xuefeng, LIU Hui. Analysis for Measurement Reliability and Maximum Permissible Error of Measuring Equipment[J]. China Measurement Technology, 2006, 32(1):14-18. 
[4] TAO Juchun, WU Jianmin. New Study on Determining the Weight of Index in Synthetic Weighted Mark Method[J]. Systems Engineering-Theory and Practice, 2001(8):43-48.

[5] XU Fuyong, SHEN Jian, LI Jianying. Study and Application of Comprehensive Assessment Mehod for Network Security[J]. Computer Engineering and Design, 2006, 27(8): 1398-1400.

[6] JJF1139-2005, Principle and Method for Determination Verification Period of Measuring Instruments [S]. 2-3.

[7] ZENG Shengkui, FENG Qiang, MA Jiming, et al. Reliability Design and Analysis[M]. Beijing: National Defense Industry Press, 2013. 68-69.

[8] WANG Xiaocong, BAI Xiaochen, LI Bo et al. Network Security Evaluation Method Based on Two-tuple Ringuistic. Computer and Digital Engineering. 2015, 43(2): 269-272.

[9] WU Zhengyang. Evaluation Method for Graduate Student Advisor Based on Two-tuple Linguistic[J]. Journal of Guangzhou University: Natural Science Edition, 2011(5): 90-94.

[10] ZHANG Yaolei, QI Yong. Method for Establishing Verification Period of Electronic Measuring Equipment Based on Reliability Model[J]. Theory and Practice, 2009,29(1):6-8.

[11] SHENG Zhou, XIE Shiqian, PAN Chengyi. Probability and Statistics[M]. Beijing: Hgiher education press, 2008. $42-46$. 